\title{
Model boson-fermion mixture within the self-consistent-field approximation
}

\author{
I. Al-Hayek \\ Department of Physics, Middle East Technical University, 06531 Ankara, Turkey \\ B. Tanatar \\ Department of Physics, Bilkent University, Bilkent, 06533 Ankara, Turkey
}

(Received 16 February 1999)

\begin{abstract}
We study the ground state properties of a boson-fermion mixture interacting via a hard-core repulsive potential with an attractive tail at zero temperature. We use the self-consistent field approach to calculate the ground state partial structure factors and the effective interactions between the species. We compare our results with the experimental data on liquid ${ }^{3} \mathrm{He}-{ }^{4} \mathrm{He}$ mixtures to find qualitative agreement. Collective modes and dynamic structure factors for the mixture are also discussed. [S0163-1829(99)08837-2]
\end{abstract}

\section{INTRODUCTION}

About a decade ago $\mathrm{Ng}$ and Singwi ${ }^{1,2}$ in a series of papers have studied a model Fermi liquid interacting via a hard-core repulsive potential and an attractive tail within the selfconsistent field approach. This simple model remarkably reproduced some key features of both the normal and spinpolarized liquid ${ }^{3} \mathrm{He}$ providing insight into the nature of strongly coupled Fermi systems. A similar investigation for a two-dimensional Fermi liquid was reported by da Silveira $e t$ $a l .^{3}$ These calculations along with some earlier reports ${ }^{4}$ have shown that the self-consistent field method of Singwi, Tosi, Land, and Sjölander ${ }^{5}$ (STLS) originally devised to treat the short-range correlation effects in Coulomb liquids (interacting via the long-range $1 / r$ potential) is also capable of handling systems interacting via short-range potentials. A qualitative agreement was found between the calculated and the experimental results. Recently, Nafari and Doroudi ${ }^{6}$ have used the realistic inter-atomic potential to study the ground state properties of liquid ${ }^{3} \mathrm{He}$ (in three and two-dimensions) within the STLS scheme, improving the level of agreement with experiments.

In this work we apply the method of $\mathrm{Ng}$ and Singwi ${ }^{1,2}$ to a boson-fermion mixture interacting via a repulsive hardcore potential. There are several motivations to study the two-component (boson-fermion) extension of the selfconsistent field approximation. First, the bipolaron model of superconductivity lends itself to an interpretation of having systems with heavy fermions based on the existence of a heavy Bose gas together with a light Fermi component. ${ }^{7}$ It is of interest to study the dielectric properties of such a twocomponent plasma. Secondly, a boson-fermion mixture of atomic gases in trap potentials ${ }^{8}$ is of recent interest because it provides a testing ground for interaction and statistical effects. Finally, a dilute solution of ${ }^{3} \mathrm{He}$ atoms in liquid ${ }^{4} \mathrm{He}$ form a fascinating quantum liquid as an example of interacting boson-fermion mixture. We can examine the present relatively simple model as applied to liquid ${ }^{3} \mathrm{He}-{ }^{4} \mathrm{He}$ mixtures to gain insight in this strongly interacting system. There has been many attempts to understand the ground state energy, correlation functions, collective excitations and singleparticle properties of this novel quantum fluid ranging from phenomenological theories ${ }^{9,10}$ to microscopic approaches ${ }^{11-14}$ and Monte Carlo simulations. ${ }^{15}$ Neutron scattering ${ }^{16-19}$ and $\mathrm{x}$-ray scattering ${ }^{20}$ experiments provide us with the information on ground state correlations.

Our primary aim in this work is to see how well the ground state properties of a boson-fermion mixture, and in particular liquid ${ }^{3} \mathrm{He}-{ }^{4} \mathrm{He}$ mixtures are described within the STLS approximation scheme. For this purpose we employ a hardcore repulsive potential with an attractive tail. Even though the model potential is far too simplistic our approach is microscopic in that the realistic helium potential can be incorporated as was done in the previous works. ${ }^{6}$ The selfconsistent field method (or the STLS approximation) renormalizes the bare hardcore potentials to yield reasonable ground state structure factors. We also study the effect of an attractive tail in the bare potential on the partial static structure factors, effective interactions, and collective excitation modes. We find that the STLS method provides a reasonable qualitative description of liquid ${ }^{3} \mathrm{He}-{ }^{4} \mathrm{He}$ mixtures which may be useful in the analysis of static and dynamical properties.

The rest of this paper is organized as follows. In the next section we outline the formulation of the STLS method in application to boson-fermion mixtures. In Sec. III we specialize to the liquid ${ }^{3} \mathrm{He}-{ }^{4} \mathrm{He}$ mixtures and present our results for the static structure factors and the effective interactions. We discuss the effects of an attractive tail on the structure factors in Sec. IV. The collective excitations of the liquid ${ }^{3} \mathrm{He}-{ }^{4} \mathrm{He}$ mixture within our model are analyzed in Sec. V. We calculate the dynamic structure factor which is of experimental relevance in Sec. VI. We conclude with a brief discussion and a summary of our results.

\section{MODEL AND THEORY}

The multicomponent generalization of the STLS theory is based on the approximation that the fluctuations in the density (of a given component) within the linear response theory is written as

$$
\delta n_{\alpha}(q, \omega)=\sum_{\beta} \chi_{\alpha \beta}(q, \omega) V_{\beta}^{\mathrm{ext}},
$$


where $\tilde{\chi}$ is the density-density response matrix and $V_{\alpha}^{\text {ext }}$ is the external perturbing field. In the self-consistent field approach of Singwi et al..$^{5}$ the response of the system to an external potential is expressed as

$$
\delta n_{\alpha}=\chi_{\alpha}^{0}(q, \omega)\left[V_{\alpha}^{\mathrm{ext}}+\sum_{\beta} V_{\alpha \beta}^{\mathrm{eff}}(q) \delta n_{\beta}\right],
$$

where $\chi_{\alpha}^{0}(q, \omega)$ is the response of the noninteracting $\alpha$ th component. Combining the above equations, we obtain the STLS expression for the density-density response function of the multicomponent system

$$
\chi_{\alpha \beta}^{-1}(q, \omega)=\left[\chi_{\alpha}^{0}(q, \omega)\right]^{-1} \delta_{\alpha \beta}-V_{\alpha \beta}^{\mathrm{eff}}(q) .
$$

The effective interparticle interactions within the STLS scheme are related to the pair-distribution functions $g_{i j}(r)$ through $^{2}$

$$
V_{\alpha \beta}^{\mathrm{eff}}(r)=-\int_{r}^{\infty} d r^{\prime} g_{\alpha \beta}\left(r^{\prime}\right) \frac{d V}{d r^{\prime}},
$$

where $V(r)$ is the bare potential taken to be the same for interactions between all species. We first start by considering a hardcore potential of the form $V(r)=V_{0} \theta\left(a_{0}-r\right)$, where $a_{0}$ is the hardcore radius and $V_{0}$ is the strength of the potential (for purely hardcore potential, we let $V_{0} \rightarrow \infty$ ). The Fourier transform of the effective potential is

$$
V_{\alpha \beta}^{\mathrm{eff}}(q)=4 \pi \frac{V_{0} g_{\alpha \beta}\left(a_{0}\right)}{q^{3}}\left[\sin \left(q a_{0}\right)-q a_{0} \cos \left(q a_{0}\right)\right] .
$$

We determine the unknown quantities $g_{\alpha \beta}\left(a_{0}\right)$, using first the fluctuation-dissipation theorem

$$
S_{\alpha \beta}(q)=-\frac{1}{\pi\left(n_{\alpha} n_{\beta}\right)^{1 / 2}} \int_{0}^{\infty} d \omega \chi_{\alpha \beta}(q, i \omega),
$$

in which $\chi_{\alpha \beta}(q, \omega)$ are the density-density response functions, and then the Fourier transform relation

$$
g_{\alpha \beta}(r)=1+\frac{1}{\left(n_{\alpha} n_{\beta}\right)^{1 / 2}} \int \frac{d \mathbf{q}}{(2 \pi)^{3}} e^{i \mathbf{q} \cdot \mathbf{r}}\left[S_{\alpha \beta}(q)-\delta_{\alpha \beta}\right] \text {. }
$$

Choosing $r=a_{0}$ in the above equations one obtains a set of nonlinear equations for the unknown quantities $V_{0} g_{\alpha \beta}\left(a_{0}\right)$ which are the multicomponent generalization of the similar expressions considered by $\mathrm{Ng}$ and Singwi. ${ }^{1,2}$ The selfconsistent field method has the same general structure as the random-phase approximation (RPA) with bare interactions replaced by effective interactions. Because the effective interactions are purely static, and no self-energy effects are included in the response functions, the model leaves no room for the multiparticle effects.

\section{APPLICATION TO ${ }^{3} \mathrm{He}-{ }^{4} \mathrm{He}$ MIXTURES}

We now specialize to the system of dilute solution of ${ }^{3} \mathrm{He}$ in ${ }^{4} \mathrm{He}$. The total number of helium atoms in the sample with volume $\Omega$ is given by $N=N_{3}+N_{4}$, in terms of the number of ${ }^{3} \mathrm{He}$ and ${ }^{4} \mathrm{He}$ atoms, and the corresponding particle density

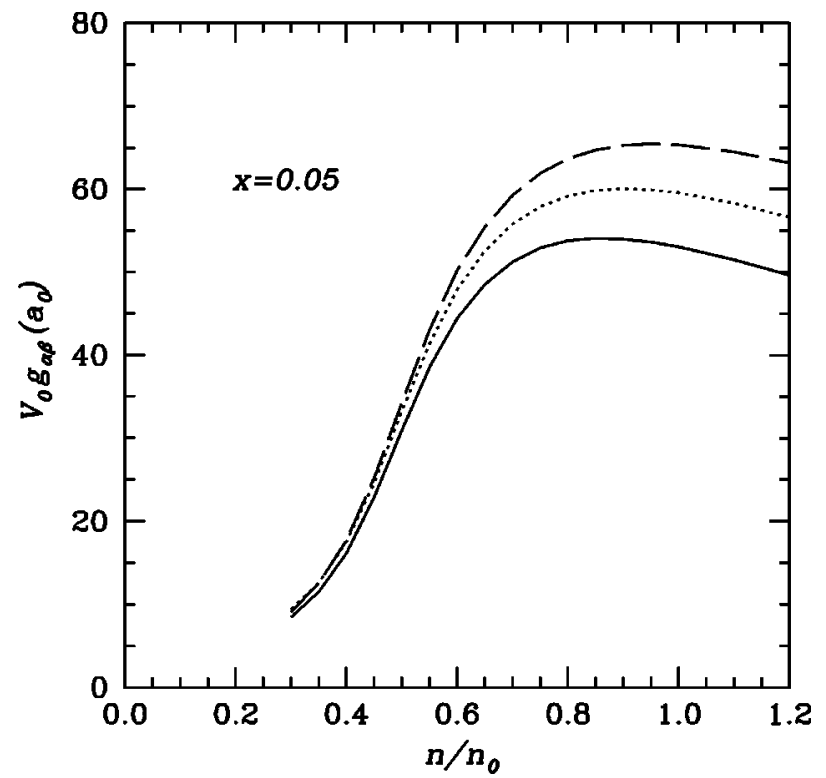

FIG. 1. The density dependence of the coefficients $V_{0} g_{\alpha \beta}\left(a_{0}\right)$ for a strictly hardcore potential $\left(V_{0} \rightarrow \infty\right)$ at the ${ }^{3} \mathrm{He}$ mole fraction $x=0.05$. The solid, dashed, and dotted lines indicate $V_{0} g_{44}\left(a_{0}\right)$, $V_{0} g_{33}\left(a_{0}\right)$, and $V_{0} g_{34}\left(a_{0}\right)$, respectively.

is $N / \Omega=n=n_{3}+n_{4}$. Denoting the fraction of ${ }^{3} \mathrm{He}$ atoms in the mixture by $x$, we have $n_{3}=x n$ and $n_{4}=(1-x) n$. We scale all lengths by the hardcore radius $a_{0}$, and the energies by the effective Rydberg $1 /\left(2 \mu a_{0}^{2}\right)$ (we take $\hbar=1$ ) where $\mu=m_{3} m_{4} /\left(m_{3}+m_{4}\right)$ is the reduced mass. For convenience the density is expressed in terms of $n_{0}=3 /\left(4 \pi a_{0}^{3}\right)$. For ${ }^{3} \mathrm{He}$ atoms in the mixture we define the Fermi wave vector $k_{F} a_{0}=\left[(9 \pi / 4) x\left(n / n_{0}\right)\right]^{1 / 3}$.

We have solved the above set of equations for the unknown parameters $V_{0} g_{\alpha \beta}\left(a_{0}\right)$ in the limit $V_{0} \rightarrow \infty$ (purely hardcore potential) for various densities and ${ }^{3} \mathrm{He}$ fractions. We illustrate our results for $x=0.05$ in Fig. 1. The density dependence of $V_{0} g_{\alpha \beta}\left(a_{0}\right)$ is smooth and shows a broad peak around $n / n_{0} \sim 0.8$. Although at low density all coefficients $V_{0} g_{\alpha \beta}\left(a_{0}\right)$ seem to vanish, around the peak region we have $V_{0} g_{33}\left(a_{0}\right)>V_{0} g_{34}\left(a_{0}\right)>V_{0} g_{44}\left(a_{0}\right)$. The behavior of $V_{0} g_{\alpha \beta}\left(a_{0}\right)$ is very similar for other values of the ${ }^{3} \mathrm{He}$ fraction in the range $0.001<x<0.1$. In the case of normal and spin-polarized fluid ${ }^{3} \mathrm{He}, \mathrm{Ng}$ and Singwi ${ }^{1,2}$ were not able to find convergent solutions to the nonlinear equations beyond a certain density and suggested the interpretation of the freezing transition. In our calculations we were able to obtain solutions for a wide range of densities. To relate our dimensionless results to the physical situation, we take $a_{0}$ $\approx 2.2 \AA$ and obtain $n_{0} \approx 0.0224 \AA^{-3}$ which is close to the equilibrium density. Thus, physical quantities calculated for the densities $n / n_{0} \sim 0.8-1.1$ should be reasonable when comparison with experiments is made.

In Fig. 2 we show our results for the static structure factors $S_{\alpha \beta}(q)$ for the ${ }^{3} \mathrm{He}-{ }^{4} \mathrm{He}$ mixture. The general behavior of the structure factors at $x=0.05$ and $n / n_{0}=1$ are depicted in Fig. 2(a). We observe that all components have qualitatively reasonable behavior compared to the more sophisticated calculations and experimental results. Because of the diluteness of the ${ }^{3} \mathrm{He}$ the resulting $S_{33}(q)$ shows very little structure and it is very different from the static structure 

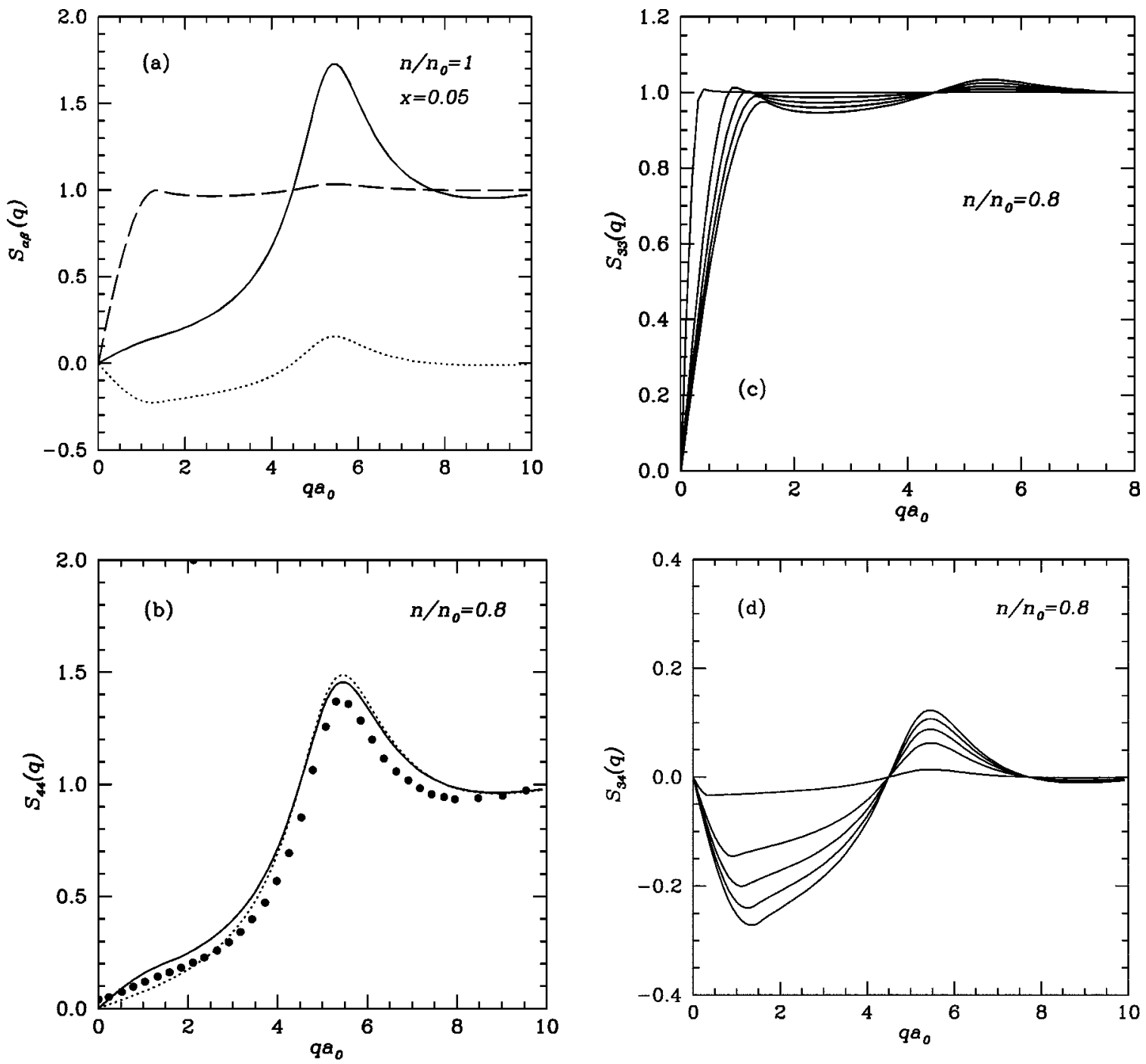

FIG. 2. (a) The partial static structure factors for liquid ${ }^{3} \mathrm{He}-{ }^{4} \mathrm{He}$ mixture at $x=0.05$ and $n / n_{0}=1$. The solid, dashed, and dot-dashed lines indicate $S_{44}(q), S_{33}(q)$, and $S_{34}(q)$, respectively. (b) $S_{44}(q)$ at $n / n_{0}=0.8$ and $x=0.08$ (solid line) and $x=0.001$ (dotted line). The filled circles are the experimental data of Svensson et al. (Ref. 21). (c) $S_{33}(q)$ at $n / n_{0}=0.8$ for $x=0.001,0.02,0.04,0.06$, and 0.08 (from left to right). (d) $S_{34}(q)$ at $n / n_{0}=0.8$ for $x=0.001,0.02,0.04,0.06$, and 0.08 (from top to bottom).

factor of pure liquid ${ }^{3} \mathrm{He}$. $S_{44}(q)$ exhibits a broad peak around $q a_{0} \approx 5.5$ (i.e., $q \sim 2.5 \AA^{-1}$ ). The peak height increases with increasing density but the position of the peak remains unchanged. We plot $S_{44}(q)$ for $x=0.001$ (dotted line) and $x=0.08$ (solid line) at a lower density $n / n_{0}=0.8$ in Fig. 2(b). We observe that with increasing ${ }^{3} \mathrm{He}$ fraction the peak value of $S_{44}(q)$ slightly decreases and the long wavelength $(q \rightarrow 0)$ behavior is modified. Also shown in the same figure is the experimental data of Svennson et al. ${ }^{21}$ for pure ${ }^{4} \mathrm{He}$. The agreement is rather good for the low ${ }^{3} \mathrm{He}$ concentration $(x=0.001)$ result. The dependence of $S_{33}(q)$ on the ${ }^{3} \mathrm{He}$ fraction is shown in Fig. 2(c). As $x$ increases there appears to be more structure in $S_{33}(q)$. If we use the decomposition $S_{33}(q)=S_{0}(q)\left[1+\Gamma_{33}(q) S_{0}(q)\right]$ where $S_{0}(q)$ is the noninteracting structure factor for fermions and $\Gamma_{33}(q)$ is the correlation part, we can relate the observed concentration dependence of $S_{33}(q)$ to the correlation effects. Finally, the structure factor $S_{34}(q)$ arising from interactions between the species is shown in Fig. 2(d) and the overall size monotonically increases. Writing $S_{34}(q)=\Gamma_{34}(q) S_{0}(q)$, we note that the correlation part $\Gamma_{33}(q)$ explicitly depends on $n_{3}^{1 / 2}$ and is responsible for the observed behavior of $S_{34}(q)$. These results are in very good qualitative agreement with those of more sophisticated theoretical calculations. ${ }^{11-13}$

Once the parameters $V_{0} g_{\alpha \beta}\left(a_{0}\right)$ are known we immediately obtain the resulting effective interactions $V_{\alpha \beta}^{\text {eff }}(q)$ within our model. Figure 3 displays the effective interactions between the ${ }^{3} \mathrm{He}$ and ${ }^{4} \mathrm{He}$ atoms for two different ${ }^{3} \mathrm{He}$ concentrations at $n / n_{0}=0.8$. The interesting feature is that the bare hardcore potential is renormalized within the selfconsistent field approximation to yield a softcore and an attractive part. It appears that the concentration dependence of $V_{\alpha \beta}^{\mathrm{eff}}(q)$ is not very strong.

\section{EFFECTS OF AN ATTRACTIVE TAIL}

The realistic potential between the helium atoms does not only have a steep hardcore but it also has an attractive tail. To this purpose it is possible to consider a model potential 


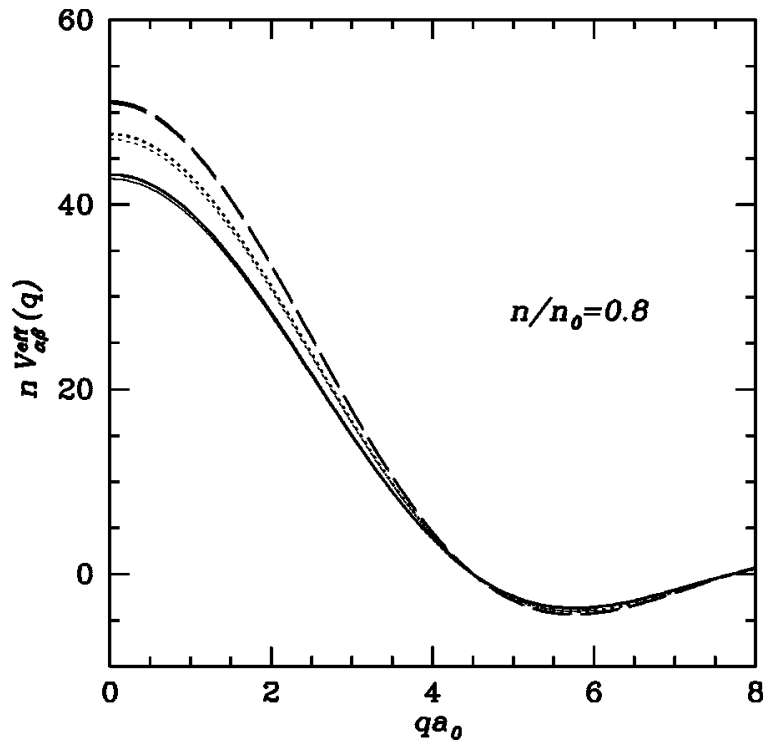

FIG. 3. The effective interactions $V_{44}^{\mathrm{eff}}(q)$ (solid), $V_{33}^{\mathrm{eff}}(q)$ (dashed), and $V_{34}^{\text {eff }}(q)$ (dotted) for liquid ${ }^{3} \mathrm{He}-{ }^{4} \mathrm{He}$ mixture at $n / n_{0}$ $=0.8$. The thick and thin lines indicate $x=0.01$ and $x=0.001$, respectively.

$$
V(r)= \begin{cases}V_{0}, & r<a_{0}, \\ -\epsilon, & a_{0}<r<a_{1}, \\ 0, & a_{1}<r,\end{cases}
$$

which yields the effective interactions

$$
\begin{aligned}
V_{\alpha \beta}^{\mathrm{eff}}(q) & =\frac{4 \pi}{q^{3}}\left(V_{0}+\epsilon\right) g_{\alpha \beta}\left(a_{0}\right)\left[\sin \left(q a_{0}\right)-q a_{0} \cos \left(q a_{0}\right)\right] \\
& -\frac{4 \pi}{q^{3}} \epsilon g_{\alpha \beta}\left(a_{1}\right)\left[\sin \left(q a_{1}\right)-q a_{1} \cos \left(q a_{1}\right)\right]
\end{aligned}
$$

where $\epsilon g_{\alpha \beta}\left(a_{1}\right)$ are extra unknown parameters to be determined. The number of equations to be self-consistently solved in this case are doubled. Because of the increased level of difficulty the minimization procedure for six nonlinear equations is slower. Furthermore, the parameters entering the problem such as $x, n / n_{0}, a_{1} / a_{0}$, and $\epsilon$ make a systematic calculation rather laborious. Therefore, we only assessed the qualitative changes occurring when an attractive tail to the bare interaction is included. The earlier calculations of $\mathrm{Ng}$ and Singwi ${ }^{1,2}$ have shown that the effect of the attractive part of the potential is quite important for pure liquid ${ }^{3} \mathrm{He}$. In Fig. 4 , we compare the partial structure factors $S_{\alpha \beta}(q)$ for liquid ${ }^{3} \mathrm{He}-{ }^{4} \mathrm{He}$ mixture with and without an attractive tail. We choose the well depth to be $\epsilon \approx 5 \mathrm{~K}$, and $a_{1} / a_{0}=2$. We observe that $S_{33}(q)$ and $S_{44}(q)$ remain largely unchanged, but the peak position of $S_{44}(q)$ is shifted towards a higher $q$ value and the peak height increases. The attractive part of the potential does not influence the resulting effective interactions $V_{\alpha \beta}^{\text {eff }}(q)$ very dramatically, only the long wavelength values are somewhat increased. Similar conclusions were also reached by $\mathrm{Ng}$ and Singwi ${ }^{1,2}$ in their study of pure ${ }^{3} \mathrm{He}$.

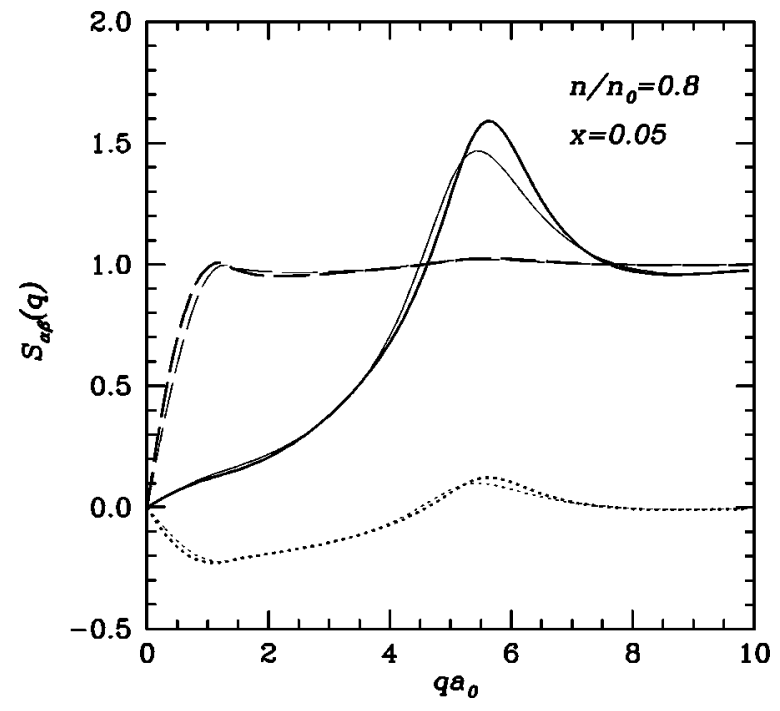

FIG. 4. The comparison of partial structure factors $S_{\alpha \beta}(q)$ in liquid ${ }^{3} \mathrm{He}-{ }^{4} \mathrm{He}$ mixture with (thick lines) and without (thin lines) attractive tail in the bare potential. Solid, dashed, and dotted lines indicate $S_{44}(q), S_{33}(q)$, and $S_{34}(q)$, respectively. We took $\epsilon$ $\approx 5 \mathrm{~K}$ and $a_{1} / a_{0}=2$.

\section{COLLECTIVE MODES}

The collective excitations are determined by solving for the roots of the determinant of the dynamic response matrix

$$
\begin{gathered}
1-V_{33}^{\mathrm{eff}}(q) \chi_{3}^{0}(q, \omega)-V_{44}^{\mathrm{eff}}(q) \chi_{4}^{0}(q, \omega)+\left\{V_{33}^{\mathrm{eff}}(q) V_{44}^{\mathrm{eff}}(q)\right. \\
\left.-\left[V_{34}^{\mathrm{eff}}(q)\right]^{2}\right\} \chi_{3}^{0}(q, \omega) \chi_{4}^{0}(q, \omega)=0 .
\end{gathered}
$$

We first look at the collective excitations of the liquid ${ }^{3} \mathrm{He}-{ }^{4} \mathrm{He}$ mixture within the mean-spherical approximation ${ }^{11}$ (MSA) for the ${ }^{3} \mathrm{He}$ component. In the MSA, the particlehole continuum and the collective mode of a Fermi system (described by the usual Lindhard function) is replaced by a single effective collective mode excitation. More specifically, the noninteracting response of ${ }^{3} \mathrm{He}$ atoms is given by

$$
\chi_{3, \mathrm{MSA}}^{0}(q, \omega)=\frac{2 n_{3} \epsilon_{q}^{(3)}}{(\omega+i \eta)^{2}-\left[\epsilon_{q}^{(3)} / S_{0}(q)\right]^{2}},
$$

where $\epsilon_{q}^{(3)}=q^{2} / 2 m_{3}$ and $S_{0}(q)$ is the Hartree-Fock static structure factor. Using the response function of the noninteracting Bose systems given by

$$
\chi_{4}^{0}(q, \omega)=\frac{2 n_{4} \epsilon_{q}^{(4)}}{(\omega+i \eta)^{2}-\left[\epsilon_{q}^{(4)}\right]^{2}},
$$

in Eq. (10), we obtain the collective mode energies

$$
\omega_{1,2}(q)=\left[\frac{1}{2}\left(\psi_{33}+\psi_{44}\right) \pm \frac{1}{2}\left[\left(\psi_{33}-\psi_{44}\right)^{2}+4 \psi_{34}\right]^{1 / 2}\right]^{1 / 2},
$$

where $\quad \psi_{33}=\left[\epsilon_{q}^{(3)} / S_{0}(q)\right]^{2}+2 n_{3} \epsilon_{q}^{(3)} V_{33}^{\mathrm{eff}}, \quad \psi_{44}=\left[\epsilon_{q}^{(4)}\right]^{2}$ $+2 n_{4} \epsilon_{q}^{(4)} V_{44}^{\text {eff }}$, and $\psi_{34}=2 n_{3} \epsilon_{q}^{(3)} 2 n_{4} \epsilon_{q}^{(4)}\left[V_{34}^{\text {eff }}\right]^{2}$. We note that free-particle energies $\epsilon_{q}^{(4)}=q^{2} / 2 m_{4}$ for the ${ }^{4} \mathrm{He}$ component are used in the noninteracting Bose response function, unlike the Feynman spectrum which contains the structure factor in the single-particle dispersion relation. The MSA is similar to 


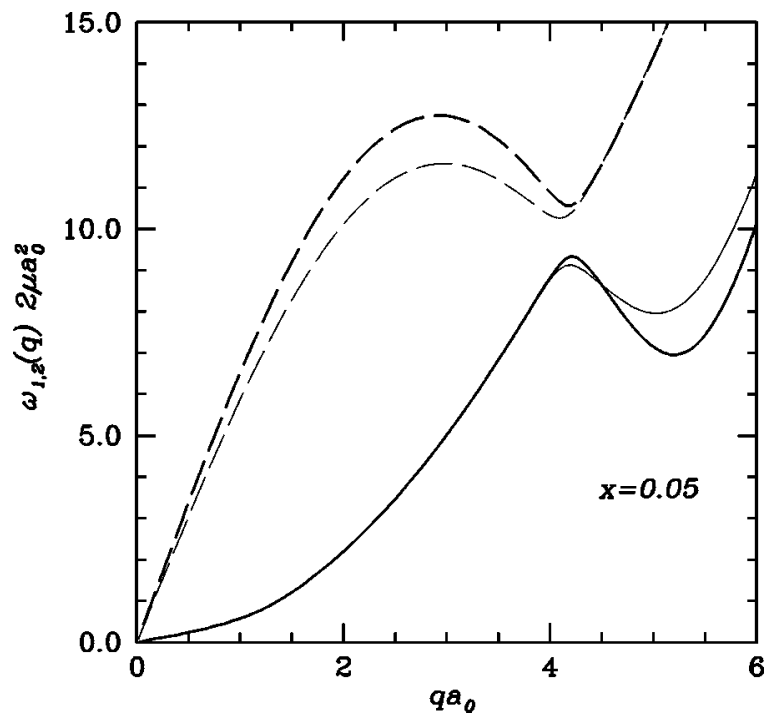

FIG. 5. The collective modes for a liquid ${ }^{3} \mathrm{He}-{ }^{4} \mathrm{He}$ mixture $(x$ $=0.05$ ) at $n / n_{0}=0.8$ (thin lines) and $n / n_{0}=1$ (thick lines) within the MSA. The upper and lower curves correspond to second sound $\left({ }^{4} \mathrm{He}\right)$ and zero sound $\left({ }^{3} \mathrm{He}\right)$ modes, respectively. The dashed lines show the effect of an attractive tail in the bare potential for $n / n_{0}$ $=1$. The thin lines are the boundaries of the particle-hole continuum.

the binary-boson approximation ${ }^{20}$ in which the ${ }^{3} \mathrm{He}$ response function $\chi_{3}^{0}(q, \omega)$ is approximated by the Bogoliubov form as for ${ }^{4} \mathrm{He}$ component. The main difference between our analytical expression for the collective modes and some other attempts ${ }^{20,22}$ is in the specification of the effective interactions $V_{\alpha \beta}^{\mathrm{eff}}(q)$. In Fig. 5 we show the collective modes within the MSA for liquid ${ }^{3} \mathrm{He}-{ }^{4} \mathrm{He}$ mixture at $x=0.05$, and two different densities. We find two discrete modes, a phonon-roton $(p r)$ branch corresponding to ${ }^{4} \mathrm{He}$ atoms (upper curves), and a second branch corresponding to ${ }^{3} \mathrm{He}$ atoms (lower curves). These modes in the small $q$ region can be identified as zeroth and second sound modes associated with the collective ${ }^{3} \mathrm{He}$ and ${ }^{4} \mathrm{He}$ excitations, respectively. ${ }^{11}$ The ${ }^{3} \mathrm{He}$ excitations show a dip similar to the roton minimum which can be regarded as a mode coupling effect. The physical content of the collective mode structure in the MSA is quite well understood. ${ }^{11} \mathrm{We}$ also point out that including an attractive tail modifies the dispersion relations slightly, around the roton minimum in the upper curve and beyond the dip structure in the lower curve.

We next turn to the full solution of Eq. (10) using the Lindhard function for $\chi_{3}^{0}(q, \omega)$. This implies that Fermi liquid effects are better treated for the ${ }^{3} \mathrm{He}$ component. The resulting dispersion of collective modes in this case are very similar to those obtained within the MSA. Again we find two distinct modes, but the second-sound mode ceases to exist as it enters the particle-hole $(p h)$ continuum. The zero-sound mode lies entirely within the $p h$ region, thus it is Landau damped. The analysis given by Krotscheck and Saarela ${ }^{11}$ and the present calculations indicate that MSA is useful in studying the collective modes in mixtures, limited only by the underlying generalized RPA.

\section{DYNAMIC STRUCTURE FACTOR}

Most of our understanding of the dynamical properties and interaction effects in liquid ${ }^{3} \mathrm{He}-{ }^{4} \mathrm{He}$ mixtures is derived from the neutron scattering experiments ${ }^{16-19}$ measuring the dynamic structure factor $S(q, \omega)$. It provides information on the density-density (and also spin-density in the case of ${ }^{3} \mathrm{He}$ component) response of the system and is useful in identifying the elementary excitations. Theoretical calculations of the dynamic structure factor have made use of phenomenological and microscopic approaches. ${ }^{23-26}$ The observed total dynamic structure factor can be separated into four terms ${ }^{27}$

$$
\begin{aligned}
S(q, \omega)= & (1-x) S_{44}(q, \omega)+2\left[x(1-x) \frac{\sigma_{3}^{c}}{\sigma_{4}^{c}}\right]^{1 / 2} S_{34}(q, \omega) \\
& +x \frac{\sigma_{3}^{c}}{\sigma_{4}^{c}}\left[S_{33}(q, \omega)+\frac{\sigma_{3}^{i}}{\sigma_{3}^{c}} S_{33}^{i}(q, \omega)\right]
\end{aligned}
$$

in which $\sigma^{c}$ and $\sigma^{i}$ are the coherent and incoherent nuclear scattering cross sections and $S_{33}^{i}(q, \omega)$ is the spectrum of spin-density excitations for the ${ }^{3} \mathrm{He}$ component in the mixture. The individual dynamic structure factors are related to the response functions through the fluctuation-dissipation theorem

$$
S_{\alpha \beta}(q, \omega)=-\frac{1}{\pi\left(n_{\alpha} n_{\beta}\right)^{1 / 2}} \operatorname{Im} \chi_{\alpha \beta}(q, \omega) .
$$

In order to calculate the spin-density response $\chi_{33}^{i}(q, \omega)$ we follow $\mathrm{Ng}$ and $\mathrm{Singwi}^{2}$ to define the spin-antisymmetric effective interaction

$$
V_{33, a}^{\mathrm{eff}}(q)=4 \pi \frac{V_{0} \tilde{g}\left(a_{0}\right)}{q^{3}}\left[\sin \left(q a_{0}\right)-q a_{0} \cos \left(q a_{0}\right)\right],
$$

where $\tilde{g}(r)$ is the Fourier transform of the static spin structure factor $\widetilde{S}(q)$. It turns out that the spin correlations in the mixture are rather weak due to the low ${ }^{3} \mathrm{He}$ concentration. Similar findings were also reported by Boronat et al. ${ }^{26}$

In Fig. 6 we show the total dynamic structure factor and its three contributions $(1-x) S_{44}(q, \omega), \quad 2[x(1$ $\left.-x) \sigma_{3}^{c} / \sigma_{4}^{c}\right]^{1 / 2} S_{34}(q, \omega), \quad$ and $\quad x\left(\sigma_{3}^{c} / \sigma_{4}^{c}\right)\left[S_{33}(q, \omega)\right.$ $\left.+\left(\sigma_{3}^{i} / \sigma_{3}^{c}\right) S_{33}^{i}(q, \omega)\right]$, using the numerical values of the scattering cross sections given in Ref. 27 . The wave vector values $q a_{0}=2$ and $q a_{0}=4$, for $x=5 \%$ and $n / n_{0}=0.8$, are displayed in Fig. 6(a) and Fig. 6(b), respectively. In the case of $q a_{0}=2$, the excitations are well separated in energy. The peak around $\omega\left(2 \mu a_{0}^{2}\right) \approx 2$ corresponds to the ${ }^{3} \mathrm{He}$ particlehole excitation, and the around $\omega\left(2 \mu a_{0}^{2}\right) \approx 10$ corresponds to the ${ }^{4} \mathrm{He}$ phonon-roton excitation. However, there is a substantial contribution from the $S_{34}(q, \omega)$ term which is negative for the $p h$ excitation and positive for the $p r$ excitation. At a higher wave vector $q a_{0}=4$, the $p h$ and $p r$ excitations come closer and the resulting total $S(q, \omega)$ exhibits a single broad peak. The individual contributions to the total dynamic structure factor are indicated in Fig. 6(b). Our results show a qualitative agreement with the calculations of Weyrauch and Szprynger ${ }^{25}$ who used the polarization potential approach to calculate the various response functions for the ${ }^{3} \mathrm{He}-{ }^{4} \mathrm{He}$ mixture. As even the simple hardcore model is able to reproduce some of the salient features of the $S(q, \omega)$, we surmise that including the realistic helium potential within the STLS 

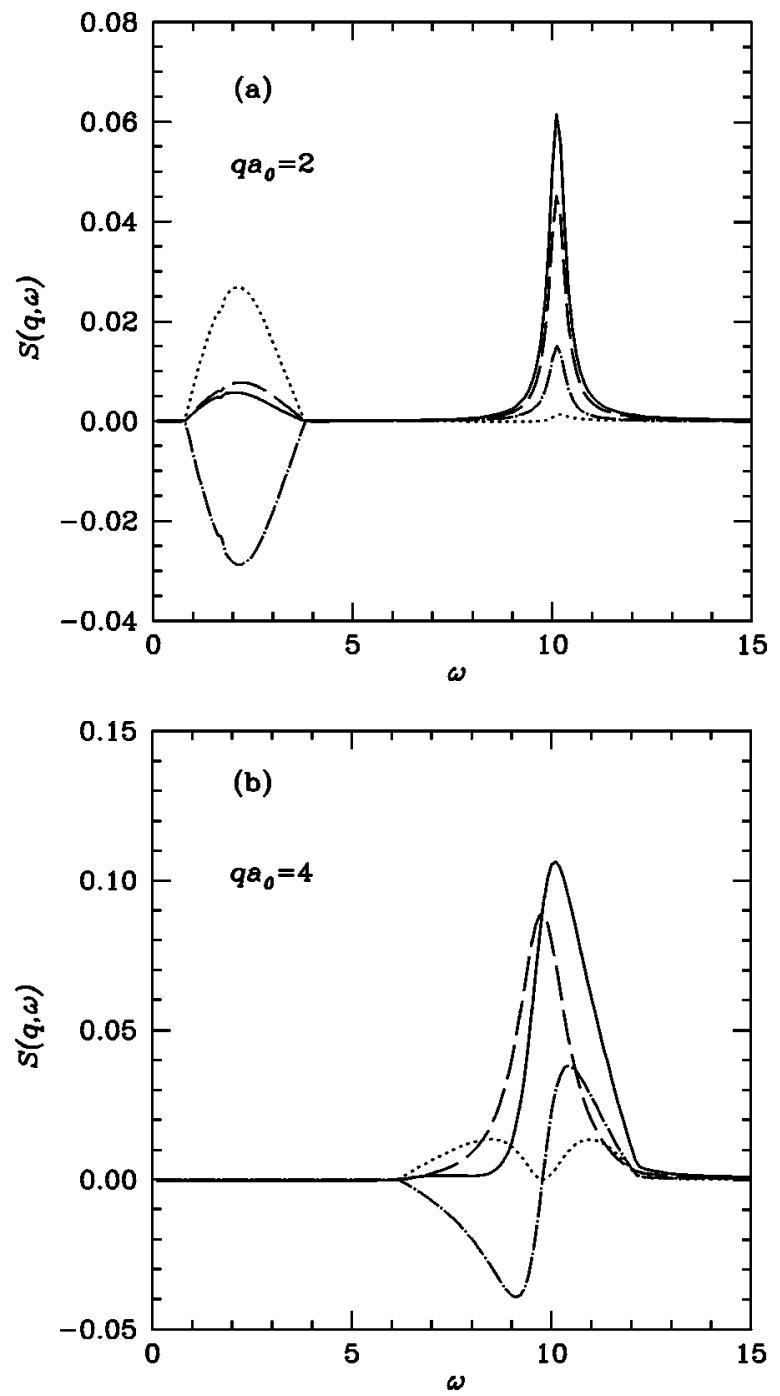

FIG. 6. The total and partial dynamic structure factors for liquid ${ }^{3} \mathrm{He}-{ }^{4} \mathrm{He}$ mixture $(x=0.05)$ at $n / n_{0}=0.8$. The solid, dashed, dotted, and dot-dashed lines indicate the total dynamic structure factor $S(q, \omega)$, and partial structure factors $S_{44}(q, \omega), S_{33}(q, \omega)$, and $S_{34}(q, \omega)$, respectively. $q a_{0}=2$ and $q a_{0}=4$ cases are shown in panels (a) and (b), respectively.

self-consistent field method might be useful in analyzing the experiments in more detail. An important shortcoming of the present approach is its omission of the multi-particle excitation effects. The calculated $S(q, \omega)$ does not, for instance, exhibit a broad contribution above the $p r$ peak. Although the positions of the peaks in $S(q, \omega)$ are not expected to change very much, the multiparticle effects should modify the peak intensities and widths which are important in determining the lifetime or damping properties of the modes.

Finally, we estimate the excitation energies $\varepsilon_{3}(q)$ and $\varepsilon_{4}(q)$ within the present model, by associating them with the $p h$ and $p r$ peaks, respectively. In Fig. 7 we compare the calculated peak positions of the total dynamic structure factor with the experimental data of Hilton et al. ${ }^{17}$ and Fåk et $a l .{ }^{18}$ Both the ${ }^{3} \mathrm{He}$ and ${ }^{4} \mathrm{He}$ excitation energies are reasonably well described by the present approach for $q$ $\lesssim 1.5 \AA^{-1}$. Similar level of agreement with the experimental data was also obtained by Fabrocini et al. ${ }^{12}$ in their correlated basis function approach, and by Weyrauch and

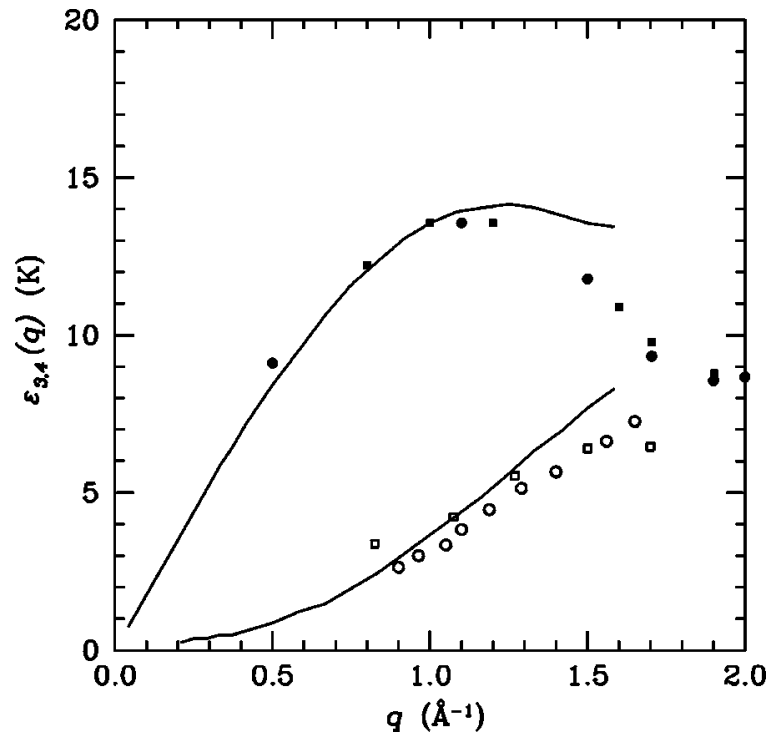

FIG. 7. The excitation energies $\varepsilon_{3}(q)$ and $\varepsilon_{4}(q)$ as calculated from the peak positions of the total $S(q, \omega)$. The circles and squares are experimental data from Ref. 18 and Ref. 17, respectively. The filled and empty symbols indicate ${ }^{4} \mathrm{He}$ and ${ }^{3} \mathrm{He}$ excitations, respectively.

Szprynger ${ }^{25}$ in their polarization potential based calculation. We note that it is somewhat surprising to find agreement with experimental data since the bare mass is used in the ${ }^{3} \mathrm{He}$ response function $\chi_{3}^{0}(q, \omega)$. However, as Fig. 6(a) illustrates the peak associated with the $p h$ excitation has significant contributions from the $S_{44}(q, \omega)$ and $S_{34}(q, \omega)$ structure factors which makes a simple free Fermi gas with effective mass interpretation difficult. A more detailed analysis involving sum rules may be useful in extracting excitation energies and Landau damping properties from the total $S(q, \omega)$.

\section{CONCLUDING REMARKS}

In this work we have extended the model Fermi liquid interacting with hardcore repulsive potential problem of $\mathrm{Ng}$ and Singwi ${ }^{1,2}$ to a mixture of boson-fermion system. The self-consistent field method with this model interaction is capable of describing qualitatively the main static and dynamic properties of liquid ${ }^{3} \mathrm{He}-{ }^{4} \mathrm{He}$ mixtures. We have found that the overall properties of the mixture are reasonably well accounted for in the range of ${ }^{3} \mathrm{He}$ mole fraction $0.01 \leq x$ $\lesssim 0.1$, and around the equilibrium density $n \approx 0.024 \AA^{-3}$. The partial static structure factors show the expected trends as a function of the ${ }^{3} \mathrm{He}$ concentration and the overall density of the system. The collective modes and dynamical structure factors reproduce qualitatively the experimental results. Our calculations of the ground state properties of ${ }^{3} \mathrm{He}-{ }^{4} \mathrm{He}$ mixtures can be extended into several directions. It is straightforward to study the mixture for which the ${ }^{3} \mathrm{He}$ component is spin-polarized ${ }^{1}$ or even with partial spin polarization. ${ }^{2}$ It should be possible to calculate the singleparticle properties of the ${ }^{3} \mathrm{He}$ component in the mixture by evaluating the self-energy using perturbation theory. This would, for instance, give information on the effective mass renormalized by interactions and the momentum distribution of the ${ }^{3} \mathrm{He}$ atoms in the system. In our numerical calcula- 
tions we have used the bare mass for ${ }^{3} \mathrm{He}$ atoms, but the experiments $^{17}$ indicate that the effective mass is $m_{3}^{*}$ $\approx 2.4 m_{3}$. For more detailed comparisons it may be necessary to take the effective mass value into account. The multiparticle effects are not taken into account within the present approach. Although the static properties are little affected, the dynamic properties such as $S(q, \omega)$ are not fully represented. Using the self-energy insertions in the response functions, it should be possible to extend the present approach to include multiparticle effects. We have based our calculations on the zero temperature STLS formalism where the ${ }^{4} \mathrm{He}$ atoms are assumed to be in the condensate. It should be possible to study the finite temperature effects by suitably modifying the response function $\chi_{44}(q, \omega)$ which also takes the particles out of the condensate into account. Finally, as our calculations demonstrate that even a hardcore potential can be treated within the STLS scheme, it would be interesting to use the realistic two-body interaction potentials between the helium atoms to make better contact with the experimental results. We expect the model calculations provided in this work will find interesting applications in other mixtures.

\section{ACKNOWLEDGMENTS}

This work was partially supported by the Scientific and Technical Research Council of Turkey (TUBITAK) under Grant No. TBAG-1662. We thank Professor G. Senatore and Dr. C. Bulutay for fruitful discussions.
${ }^{1}$ T.K. Ng and K.S. Singwi, Phys. Rev. Lett. 57, 226 (1986).

${ }^{2}$ T.K. Ng and K.S. Singwi, Phys. Rev. B 35, 1708 (1987); 35, 6683 (1987).

${ }^{3}$ H.V. da Silveira, M.H. Degani, and K.S. Singwi, Phys. Rev. B 46, 2995 (1992).

${ }^{4}$ R. Lobo and P.R. Antoniewicz, Phys. Rev. Lett. 24, 1168 (1970); R. Lobo, Phys. Rev. B 12, 2640 (1975); G. Niklasson and K.S. Singwi, Solid State Commun. 59, 575 (1986).

${ }^{5}$ K.S. Singwi, M.P. Tosi, R.H. Land, and A. Sjölander, Phys. Rev. 176, 589 (1968); K.S. Singwi and M.P. Tosi, Solid State Phys. 36, 177 (1981).

${ }^{6}$ N. Nafari and A. Doroudi, Phys. Rev. B 51, 9019 (1995); A. Doroudi, ibid. 58, 438 (1998).

${ }^{7}$ A.S. Aleksandrov and J. Ranninger, Phys. Rev. B 23, 1796 (1981); 24, 1164 (1981); A.S. Aleksandrov, J. Ranninger, and S. Robaszkiewicz, Phys. Rev. Lett. 56, 949 (1986).

${ }^{8}$ L. Vichi, M. Inguscio, S. Stringari, and G.M. Tino, J. Phys. B 31, L899 (1998); M. Amoruso, A. Minguzzi, S. Stringari, M.P. Tosi, and L. Vichi, Eur. Phys. J. D 4, 261 (1998).

${ }^{9}$ W. Hsu, D. Pines, and C.H. Aldrich, Phys. Rev. B 32, 7179 (1985); W. Hsu and D. Pines, J. Stat. Phys. 38, 273 (1985).

${ }^{10}$ M. Lücke and A. Szyprynger, Phys. Rev. B 26, 1374 (1982); A. Szyprynger and M. Lücke, ibid. 32, 4442 (1985).

${ }^{11}$ For a recent review see, E. Krotscheck and M. Saarela, Phys. Rep. 232, 1 (1993).

${ }^{12}$ A. Fabrocini, L. Vichi, F. Mazzanti, and A. Polls, Phys. Rev. B 54, 10035 (1996).
${ }^{13}$ E. Krotscheck, J. Paaso, M. Saarela, K. Schörkhuber, R. Zillich, Phys. Rev. B 58, 12282 (1998).

${ }^{14}$ E. Talbot and A. Griffin, J. Low Temp. Phys. 56, 141 (1984).

${ }^{15}$ S. Moroni and M. Boninsegni, Europhys. Lett. 40, 287 (1997).

${ }^{16}$ J.M. Rowe, D.L. Price, and G.E. Ostrowski, Phys. Rev. Lett. 31, 510 (1973).

${ }^{17}$ P.A. Hilton, R. Scherm, and W.G. Stirling, J. Low Temp. Phys. 27, 851 (1977).

${ }^{18}$ B. Fåk, K. Guckelsberger, M. Körfer, R. Scherm, and A.J. Dianoux, Phys. Rev. B 41, 8732 (1990).

${ }^{19}$ Y. Wang and P.E. Sokol, Phys. Rev. Lett. 72, 1040 (1994).

${ }^{20}$ M. Suemitsu and Y. Sawada, Phys. Lett. 71A, 71 (1979); Phys. Rev. B 25, 4593 (1982).

${ }^{21}$ E.C. Svennson, V.F. Sears, A.D.B. Woods, and P. Martel, Phys. Rev. B 21, 3638 (1980).

${ }^{22}$ T. Soda, Prog. Theor. Phys. 86, 825 (1991).

${ }^{23}$ J. Ruvalds, J. Slinkman, A.K. Rajagopal, and A. Bagchi, Phys. Rev. B 16, 2047 (1977).

${ }^{24}$ K.S. Pedersen and R.A. Cowley, J. Phys. C 16, 2671 (1983).

${ }^{25}$ M. Weyrauch and A. Szprynger, Phys. Rev. B 51, 12698 (1995).

${ }^{26}$ J. Boronat, F. Dalfovo, F. Mazzanti, and A. Polls, Phys. Rev. B 48, 7409 (1993).

${ }^{27}$ H.R. Glyde and E.C. Svensson, in Methods of Experimental Physics, edited by D.L. Price and K. Sköld (Academic, New York, 1987), Vol. 23B, p. 303. 